\title{
As cidades médias brasileiras precisam ser mais amigáveis aos morcegos, principalmente nos hotspots de biodiversidade
}

\section{Las ciudades medias de Brasil necesitan ser más amigables a los murciélagos, principalmente en los hotspots de biodiversidad}

\author{
Brazilian medium-sized cities need to be more friendly for bats, \\ mainly on biodiversity hotspots
}

\author{
Isabela Carolina Ortêncio Negri \\ isabelaortencio@gmail.com \\ Universidade Federal de Mato Grosso, UFMT, campus de Rondonópolis, MT \\ Sérgio Sebastião Negri \\ sernegri@gmail.com \\ Universidade Federal de Mato Grosso, UFMT, campus de Rondonópolis, MT
}

Fabio Angeoletto

fabio_angeoletto@yahoo.es

Universidade Federal de Mato Grosso, UFMT, campus de Rondonópolis, MT

Resumo: Atualmente mais da metade da população mundial vive em cidades e este número deve chegar a $72 \%$ até 2050. No Brasil, o processo de urbanização do território, geralmente rápido e sem planejamento, origina uma miríade de impactos ambientais. A urbanização faz declinar a riqueza e diversidade de espécies. Por outro lado, algumas espécies se adaptam aos ambientes antropogênicos pois obtêm vantagens da matriz urbana, utilizando-se de abrigo ou alimento disponíveis. Os morcegos possuem uma plasticidade ecológica que os permite estabelecerem-se em remanescentes florestais nas cidades ou diretamente no meio construído. Nesse ensaio, nós defendemos a importância das cidades médias como espaços de conservação da biodiversidade.

Palavras-chave: quirópteros; urbanização; biodiversidade urbana; ecologia urbana.

Resumen: Actualmente, más de la mitad de la población mundial vive en ciudades y se espera que este número alcance el $72 \%$ para 2050. En Brasil, el proceso de urbanización del territorio, generalmente rápido y no planificado, causa una gran cantidad de impactos ambientales. La urbanización hace que la riqueza y diversidad de las especies disminuyan. Por otro lado, algunas especies se adaptan a los ambientes antropogénicos porque obtienen ventajas de la matriz urbana, utilizando refugio o alimentos disponibles. Los murciélagos tienen una plasticidad ecológica que les permite establecerse en los restos de bosques en las ciudades o directamente en el entorno construido. En este ensayo, defendemos la importancia de las ciudades medianas como espacios para la conservación de la biodiversidad.

Palabras clave: quirópteros; urbanización; biodiversidad urbana, ecología urbana. 
Abstract: Nowadays, more than half of the world's population lives in cities and this number is expected to reach $72 \%$ by 2050 . In Brazil, the process of urbanization of the territory, usually rapid and unplanned, causes a myriad of environmental impacts. Urbanization makes the wealth and diversity of species decline. On the other hand, some species adapt to the anthropogenic environments because they obtain advantages of the urban matrix, using shelter or food available. Bats have an ecological plasticity that allows them to settle in forest remnants in the cities or directly in the built environment. In this essay, we defend the importance of medium-sized cities as spaces for the conservation of biodiversity.

Keywords: chiropterans; urbanization, urban biodiversity, urban ecology.

\section{INTRODUÇÃO}

A população mundial precisou de milhares de anos para alcançar o primeiro bilhão de habitantes. Mas, após a Segunda Guerra Mundial, seu crescimento se tornou extremamente acelerado, acompanhado do fenômeno da migração. Sua distribuição espacial também se alterou profundamente. A urbanização é a característica marcante da nova repartição geográfica da população mundial. Em 2008, mais da metade da população humana mundial vivia em cidades, e essa proporção é prevista crescer para 72\% até 2050 (ANGEOLETTO et al., 2016). Daí a metáfora de Angeoletto (2012), de Planeta Cidade.

No Brasil, em poucas décadas, dezenas de milhões de pessoas deixaram a zona rural em direção às cidades. Em 1890, a população urbana era, cerca de, 10\% do total nacional. Atualmente, $83 \%$ dos brasileiros habitam as cidades (ANGEOLETTO et al., 2018). Num primeiro ciclo, os emigrantes se dirigiram sobretudo ao Sudeste do país, atraídos pela atividade industrial nas cidades grandes, mas, a partir de 1990, teve início uma nova dinâmica de urbanização no Brasil, associada à interiorização da atividade industrial, com um aumento considerável do número de cidades médias, processo denominado por Santos (2005), como 'dissolução da metrópole', em alusão a relativa desindustrialização cidade de São Paulo.

No território brasileiro, existem 5.565 municípios, dentre estes 264 comportam cidades consideradas médias. Nestas, vivem cerca de $25 \%$ do total da população e, além disso, as cidades médias apresentam um ritmo de crescimento mais acelerado ${ }^{1}$ que as demais, sejam a grandes e/ ou pequenas (IBGE, 2010; ANGEOLETTO et al., 2016).

A dinâmica populacional está intrinsecamente vinculada às atividades socioeconômicas, consoantes a diferentes períodos históricos, que resultaram em impactos ambientais de várias escalas territoriais. O processo acelerado de urbanização, usualmente, está associado ao desmatamento de vastas áreas e à posterior produção e circulação de mercadorias em ritmo igualmente veloz. A urbanização acarreta uma transformação do ambiente natural em uma matriz artificial de concreto e asfalto (ÁVILA-FLÓREZ, 2005) a tal extremo que as áreas urbanas são consideradas como zonas mais alteradas do planeta (KOZLOV, 1996;

1 Por exemplo, entre 1991 e 2000, a taxa anual de crescimento das cidades médias brasileiras foi de 2,4\%, enquanto as cidades grandes e metrópoles cresceram apenas 1,6\% (IBGE, 2000). 
COLLINS et al., 2000; GERMAINE; WAKELING, 2001).Em um sentido amplo, a expressão urbanização, significa a conversão do solo em ambientes urbanos e, por estes, não definimos só a área das cidades, mas, também, as áreas contíguas a estas, apropriadas por elas, e que proveem energias materiais e absorvem seus dejetos (ANGEOLETTO et al., 2017).

As cidades estão, portanto, no centro dos debates sobre mudanças climáticas, conservação da biodiversidade e bem-estar dos primatas humamos (GRIMM et al., 2008; HOORNWEG; SUGAR; TREJOS GÓMEZ, 2011; NEWMAN, 2006; HODSON; MARVIN, 2009; GLAESER, 2011; ANGEOLETTO et al., 2018; RUMBLE et al., 2019).

Os efeitos da urbanização dependem de sua natureza, das características da paisagem que conforma a cidade e de sua escala (DREW, 1989; CLERGEAU et al., 1998; HARDY; DENNIS, 1999). A persistente tendência de declínio da riqueza e diversidade das espécies representa alguns dos resultados do processo de urbanização (MCKINNEY, 2008,2002), assim como o aumento do grau de homogeneização biótica para a maioria dos táxons, gerando um impacto considerável na biodiversidade (LOCKWOOD et al., 2000; MCKINNEY; LOCKWOOD, 1999; MCDONALD; MARTY; FORMAN, 2008).

Por outro lado, algumas espécies podem ser favorecidas pelo processo de urbanização, porque elas se adaptam muito bem em paisagens heterogêneas, obtendo vantagens nesses ambientes (GILBERT, 1989; MAGLE et al., 2012), seja se beneficiando, por exemplo, da maior temperatura nas áreas centrais urbanas (COSTANZA et al., 2014), ou mesmo abrigando-se nas edificações, para se proteger de grandes predadores (BAKER; HARRIS, 2007).

\section{INFORMAÇÕES RELEVANTES SOBRE A ORDEM CHIROPTERA}

Os morcegos são os únicos mamíferos adaptados ao voo e ocupam o ambiente terrestre de todos os continentes. Eles estão ausentes apenas em algumas ilhas do Oceano Pacífico (por serem muito isoladas do continente) em regiões muito frias, como as calotas polares ou em regiões de altitude extrema (LAURINDO; NOVAES, 2015). Os morcegos constituem a segunda maior ordem de mamíferos, sendo superados apenas pelos roedores (SIMMONS, 2005), compreendendo 20 famílias e 1300 espécies conhecidas (TSANG et al., 2016; FENTON; SIMMONS 2014), 20\% de todas as espécies conhecidas de mamíferos (KOOPMAN, 1993). As seis famílias consideradas endêmicas na América do Sul e no Brasil são a Noctilionidae, Furipteridae, Natalidae, Phyllostomidae, Tyropteridae e a Mormoopidae, e duas apresentam ampla distribuição mundial, Molossidae e Vespertilionidae (KOOPMAN, 1981; TIRIRA, 1998). Atualmente, são conhecidas no Brasil 178 espécies de morcegos pertencentes a nove famílias e 68 gêneros - o Brasil é o segundo país do mundo em diversidade de quirópteros (NOGUEIRA et al., 2014).

Morcegos são animais de hábitos noturnos, que, apesar de não serem cegos, apresentam poucos cones em sua retina e utilizam a eco-localização para sua orientação (NEUWEILER, 2000), permitindo evitar obstáculos na escuridão, localizar e capturar suas presas (HICKMAN; ROBERTS; LARSON, 2004). Também, existem três espécies hematófagas restritas à região neotropical (BRASS, 1994). Os quirópteros são também importantes 
em estudos relacionados à sanidade animal e saúde pública, por serem considerados reservatórios de várias zoonoses, dentre elas a raiva (CUNHA et al., 2006). O conhecimento de sua diversidade, ecologia e biologia é importante para diversos estudos sobre manejo de suas populações, pois estes mamíferos voadores também atuam como bioindicadores de distúrbios nos ecossistemas (MEDELLIN; EQUIHUA; AMIN, 2000).

Os morcegos desempenham importantes funções dentro do ecossistema, devido às suas diversas interações ecológicas (KUNZ et al., 2011), consomem insetos e outros artrópodes, pequenos vertebrados, sangue, flores, folhas, frutos e sementes, uma dieta muito diversificada (PERACCHI et al., 2006), desta maneiraatuam como controladores de populações de insetos (KUNZ; WHITAKER; WADANOLI, 1995)., dispersores de sementes (RIDLEY, 1930; LOPEZ; VAUGHAN, 2004; SATO; PASSOS; NOGUEIRA, 2008) e polinizadores (BAKER, 1961; SILVA; PERACCHI, 1995; MARTINS; GRIBEL, 2007), além de participarem de outros processos ecológicos, relacionados à predação e à hematofagia (REIS et al., 2007).

Os quirópteros são reconhecidos pela qualidade da dispersão que promovem, geralmente consumindo frutos maduros, os quais transportam para longe da planta matriz, e defecam sementes em voo, frequentemente sobre áreas abertas, propícias à germinação (LOBOVA et al., 2009). Uma grande parcela desses animais se alimenta de frutos de plantas pioneiras (GALINDO GONZÁLEZ et al., 2009), sendo, importantes nos estágios iniciais da sucessão florestal (MUSCARELLA; FLEMING, 2007). Segundo Mello (2007), estas dispersões a longas distâncias favorecem o aumento da variabilidade genética das futuras plantas, pois, possibilitam o cruzamento entre vegetais de populações diferentes. Globalmente, segundo Wilson (1991), a região Neotropical se destaca como a mais atrativa para espécies de morcegos frugívoros, pois já foram registradas interações com pelo menos 546 espécies de plantas, em 191 gêneros e 62 famílias (LOBOVA et al., 2009). Algumas espécies de plantas dependem exclusivamente dos morcegos para sua dispersão (SAZIMA; BUZATO; SAZIMA, 2003; THIES; KALKO, 2004). No Brasil 189 espécies de plantas são utilizadas por morcegos (FABIÁN et al., 2008), com destaque para os animais da família Phyllostomidae, considerados os maiores responsáveis pela regeneração das florestas na região Neotropical (HUTSON et al., 2001).

Segundo Peracchi (2006), os morcegos ocupam posição de destaque no controle de populações de insetos, incluindo espécies prejudiciais às lavouras. Os quirópteros pertencentes às famílias Vespertilionidae e Molossidae, de hábito alimentar insetívoro, capturam de seis a vinte gramas de insetos por noite, tanto no meio rural quanto no urbano (FABIÁN et al., 1990; MCCRACKEN et al., 2001).

Os morcegos também são vetores de algumas doenças. Das 178 espécies conhecidas no território nacional, 41 já foram registradas como positivas para raiva. Destas, 25 foram observadas explorando refúgios em habitações humanas ou no entorno delas, com provável acesso a cães e gatos (SODRÉ; GAMA; SILVA, 2010). Essa proximidade de morcegos com os seres humanos e animais de estimação podem elevar o risco de ocorrência contaminações. Os morcegos, independentemente de seu hábito alimentar, podem hospedar e transmitir o vírus da raiva direta ou indiretamente a seres humanos ou animais. Mesmo no caso de 
morcegos não-hematófagos, esta transmissão pode ocorrer de forma acidental (UIEDA; HARMANI; SILVA, 1995).

A doença mais comumente associada aos morcegos é a raiva. No entanto, os morcegos infectados representam apenas $1 \%$ do total de indivíduos presentes no território brasileiro, enquanto isso, nas grandes capitais brasileiras, os índices de positividade para raiva em morcegos são ainda mais baixos, oscilando entre 0,5\% e 0,8\% do total, o que corresponde aos indicadores de normalidade estabelecidos pela Organização Mundial de Saúde (PACHECO et al., 2010).Outra doença associada aos morcegos, apesar de pouco conhecida, é a histoplasmose. Potencialmente presente em locais pouco ventilados e com acúmulo de guano (fezes de morcego) podem promover a suspensão de esporos de fungos como o Histoplasma capsulatum (DARLING, 1906), causador da enfermidade (ESBÉRARD et al., 1999; FERREIRA; BORGES, 2009; PACHECO et al., 2010).

Além de doenças, a presença de morcegos pode causar uma série de transtornos, seja em áreas residenciais, bem como em edificações comerciais, industriais e de serviços, quando essas construções são ocupadas pelos quirópteros. Seja pelo odor forte, sobretudo nos dias de calor intenso, oriundo de sua urina e/ou acúmulo de guano, bem como pelos ruídos que causam quando vocalizam (PEDRO, 1998). Dentro de construções, os morcegos também podem causar a contaminação de alimentos e da água armazenada nas caixas d'água, se não estiverem convenientemente fechadas (ESBÉRARD et al., 1999; REIS; DE LIMA; PERACCHI, 2006; SCHNEIDER, et al., 2009; CARVALHO et al., 2011).

\section{MORCEGOS EM ECOSSISTEMAS URBANOS}

Uma das maiores causas da diminuição da população de morcegos é a perda de hábitat e de recursos por causa da urbanização e agricultura (KRAUEL; LEBUHN, 2016; BORDER et al., 2017; WORDLEY et al., 2017). Não é surpreendente que muitos estudos tenham demonstrado que essas formas de perda de hábitat, fragmentação e modificação tem um impacto negativo na atividade dos morcegos, na sua abundância e na sua diversidade (TRENTINI, 2005; FRICK et al., 2017; GONÇALVES; FSCHER; DIRZO, 2017; LINLEY 2017; KAHNONITCH; LUBIN; KORINE, 2018). Essa supressão dos habitats naturais ocasionou a extinção das espécies de quirópteros mais vulneráveis e com menor capacidade de adaptação (DEBINSKI; HOLT, 2000). Por outro lado, muitas espécies de morcegos, não necessariamente generalistas, estão se beneficiando e utilizando recursos alternativos de origem antropogênica (RODRIGUEZ-AGUILAR et al., 2017; VERDE; SILVA; CALOURO., 2018), como abrigo e alimento, edificações, plantas e iluminação noturna nas cidades (UIEDA et al., 1995; FENTON, 1992, 1997).

A densidade e a diversidade de morcegos em ecossistemas urbanos podem variar devido a diversos fatores, como, a disponibilidade de dormitórios, presença e distância entre árvores, disponibilidade de água, e conectividade entre áreas verdes por meio de corredores de árvores (CÔRTES et al., 1994; BREDT; UIEDA, 1996; FENTON, 1997; SAZIMA, 
et al., 2003; ESBÉRARD et al., 1999; ZÓRTEA; AGUIAR, 2001; LIMA, 2008; OPREA et al., 2009; MCKINNEY, 2002).

Segundo Ferreira et al. (2010), os morcegos destacam-se dentre os animais com maior capacidade de dispersão territorial, tanto no campo como na cidade, seja utilizando remanescentes de vegetação nativa, ou ambientes construídos que ofereçam abrigos e fontes de alimentos. Assim, estes indivíduos se fazem cada vez mais presentes em áreas urbanizadas. Sua maior ou menor presença nas cidades dependerá das características da malha urbana, da arquitetura dos imóveis e, também, pela dimensão e espraiamento do sítio urbano (SOARES et al., 2011).

Segundo o estudo de Estrada e Coates-Estrada (2001), devido à sua capacidade de dispersão, morcegos são menos vulneráveis à fragmentação quando comparados a outros mamíferos, pois apresentam adaptação relativamente mais fácil em ambientes alterados, e também devido à flexibilidade ecológica encontrada em algumas espécies (TADDEI, 1983; BREDT; UIEDA, 1996; BARROS; BISAGGIO; BORGS, 2006). Essa plasticidade ecológica permite que comunidades de quirópteros se estabeleçam em remanescentes florestais nas cidades ou diretamente no meio construído (REIS et al., 2006; PERINI et al., 2014; SILVA; PERINI; OLIVEIRA, 2005), uma vez que esses ambientes disponibilizam abundância e variedade de recursos alimentares, além de abrigos oportunos (BREDT; UIEDA, 1996; ESBÉRARD, 2003; LIMA, 2008; OPREA et al., 2009).

Nas cidades, ademais de morcegos frugívoros, há espécies insetívoras e fitófagas. Os morcegos fitófagos são aqueles que se beneficiam diretamente das plantas usadas na arborização, utilizando seus frutos, folhas e néctar das flores (SILVA et al., 1996; BREDT et al., 1998; SAZIMA et al., 2003). Os morcegos insetívoros também obtem suas presas em áreas urbanas com relativa facilidade, pois estas são atraídas pela iluminação pública (BREDT; UIEDA, 1996; SILVA et al., 1996), proporcionada por lâmpadas de mercúrio, largamente usadas nas ruas e avenidas (RYDELL; RACEY, 1995). Entretanto, a diversidade de morcegos insetívoros em áreas urbanas é menor do que em áreas não urbanas, como fragmentos de matas em zonas rurais (KURTA; TERAMINO, 1992).

\section{HÁ POUCAS PESQUISAS SOBRE QUIRÓPTEROS EM ECOSSISTEMAS URBANOS}

No Brasil existem poucas informações relacionadas à conservação e ecologia de quirópteros tanto em áreas urbanas quanto em ambientes naturais (PACHECO 2010; NUNES; ROCHA; CORDEIRO-ESTRELA, 2017). A maioria dos trabalhos voltados a esta temática se refere a pesquisas de impactos ambientais, inventários faunísticos e análises sobre a estrutura de assembleias (SAMPAIO et al., 2003; CLEVELAND et al., 2006; BARCLAY; BAERWALD; GRUVER, 2007; KUNZ et al., 2007; BIANCONI et al., 2008).

Estes estudos podem ser potencializados para investigar quais espécies podem se adaptar aos ecossistemas urbanos (LIMA, 2008). Seu comportamento, usos de habitats alterados, padrões de deslocamento, preferências por abrigos e reprodução, também são 
pouco estudados (MARQUES FABIÁN, 1994; MARQUES; PACHECO, 1999; SODRÉ; ESBÉRARD, 2008; UIEDA et al., 2008).

Os estudos sobre a ocorrência e distribuição espacial de morcegos têm apresentado um crescimento no Brasil nos últimos anos, mas a maioria das pesquisas se restringe a menos de 10\% de toda extensão do território nacional (BERNARD et al., 2011). A Região Sudeste se destaca como área onde é realizada a maioria dos estudos sobre morcegos (BERGALLO et al., 2003; ESBÉRARD; BERGALLO 2005; NUNES et al., 2017), enquanto o Estado do Rio de Janeiro é detentor do maior número de indivíduos coletados (BERGALLO et al., 2003; ESBÉRARD; BERGALLO, 2005) como, também, possuidor do mais longo histórico de pesquisas (PERACCHI; NOGUEIRA, 2010). Entretanto, em áreas urbanas, o número de estudos e a quantidade de morcegos coletados ainda se apresenta como insuficiente inclusive na Região Sudeste do país (PACHECO et al., 2010).

\section{COMO TORNAR RONDONÓPOLIS, UMA CIDADE INSERIDA EM UM HOTSPOT DE BIODIVERSIDADE, MAIS AMIGÁVEL AOS MORCEGOS?}

No final do XIX e no início do século XX, a região onde foi fundada a cidade de Rondonópolis era coberta por vegetação típica do Cerrado. Mas a partir da década de 1970, segundo Negri (2001), a ocupação do território de Rondonópolis e do Sudeste de Mato Grosso é marcada pela expansão de uma frente pioneira contemporânea sobre as terras de cerrado em moldes tipicamente capitalistas. Incluindo a mecanização e o uso maciço de insumos físico-químicos e biológicos no cultivo de novas culturas destinadas à exportação, sobretudo a soja, que se expandem em detrimento das culturas de gêneros alimentares e sobre a pecuária extensiva nas terras do Cerrado.

A partir desse período, a vegetação do Cerrado tem sido um dos biomas mais modificados pelas ações humanas (MYERS et al., 2000; GOLDSTEIN et al., 2008). O domínio do Cerrado é um mosaico de vegetação variando de savanas a ambientes florestais, como cerradão, vegetação ripária e galerias de florestas (COUTINHO, 2006; BATALHA; MANTOVANI, 2011).

Essa heterogeneidade no Cerrado aumenta a riqueza de espécies, dá suporte uma alta biodiversidade (MACHADO et al., 2004; KLINK; MACHADO, 2005) e muitas espécies endêmicas. Entretanto, essa fauna e flora estão ameaçadas pela expansão continuada de áreas para a agricultura, pastagens e pelo avanço de áreas urbanas (KLINK; MACHADO, 2005; SILVA et al., 2017; VACCHIANO et al., 2019), o que lhe confere o status de hotspot de biodiversidade (MYERS et al., 2000).

No Estado do Mato Grosso, a vegetação nativa do cerrado foi rapidamente substituída pelas monoculturas, particularmente pelas plantações de soja, algodão e milho, favorecidas pela topografia, no processo de mecanização. Como resultado, o cerrado remanescente é agora amplamente fragmentado e isolado (VILHENA-VIALOU, 2006; DURIGAN; SIQUEIRA; FRANCO, 2007).Diante desse quadro, a manutenção da biodiversidade do Cerrado depende, em grande parte, do conhecimento e da conservação das espécies 
que habitam remanescentes florestais em áreas urbanas e rurais (FERREIRA et al., 2010; ANGEOLETTO et al., 2018; ANGEOLETTO et al., 2019; OLIVEIRA et al., 2019; VACCHIANO et al., 2019).

No Cerrado ocorrem pelo menos 103 espécies de morcegos, que representam aproximadamente $62 \%$ da fauna de quirópteros do Brasil (AGUIAR; ZORTÉA, 2008; REIS et al., 2007), e a mais de $40 \%$ da quiropterofauna da América do Sul (MARINHOFILHO, 1996). As espécies do Cerrado brasileiro estão distribuídas em 42 gêneros e em sete das nove famílias conhecidas para a região Neotropical (MARINHO-FILHO, 1996). Entretanto, apesar da grande diversidade deste grupo e da sua importância ecológica, o conhecimento atual sobre a fauna de morcegos do Cerrado é ainda pífio (AGUIAR; ZORTÉA, 2008; NUNES et al., 2017).

Estimativas indicam que a taxa de expansão da atividade agrícola sobre as áreas nativas do Cerrado é de $3 \%$ ao ano e que, até 2030, esse bioma estará restrito a áreas legalmente protegidas (AQUINO; MIRANDA, 2008; SILVA et al., 2017; VACCHIANO et al., 2019). A extrema e acelerada degradação do Cerrado impõe às cidades inseridas nesse hotspot de biodiversidade que se reorganizem para tornarem-se mais amigáveis à diversidade biológica do bioma. Aumentar o suporte à biodiversidade urbana demanda uma maior compreensão dos diversos fatores que influem sob os quais os ecossistemas urbanos. Fatores ambientais, econômicos, culturais, urbanísticos e sociais interagem e explicam a maior ou menor capacidade de uma cidade em dar suporte à diversidade biológica.

No caso dos morcegos, existe muita resistência da sociedade em relação à sua presença em ambientes urbanos, devido ao preconceito estabelecido, decorrente de mitos e lendas que os relacionam com vampiros. Dessa forma, os morcegos são considerados perigosos e desagradáveis (PACHECO et al., 2010).

Nós estamos estudando as espécies de morcegos que estão presentes na matriz urbana de Rondonópolis. O estudo é conduzido em parceria com o People and Wildlife Research Group, da Universidade de Reading (UK). Estamos desenvolvendo um projeto que analisa o uso das piscinas por quirópteros como fonte antrópica alternativa de água, além de verificar se estas próprias alternativas hídricas também são armadilhas ecológicas para os mesmos. Em paralelo, estamos coletando dados a respeito de uso dos fragmentos urbanos de Cerrado do município de Rondonópolis por morcegos. Nós temos o objetivo de respaldar a Secretaria de Meio Ambiente de Rondonópolis, para que esses fragmentos adquiram o status de unidades de conservação municipais. Rondonópolis não é Gotham City - não na escala metropolitana. Mas esperamos contribuir para que essa cidade de porte médio engastada no Cerrado esteja repleta de morcegos. 


\section{AGRADECIMENTOS}

Ao professor Henrique Ortêncio Filho, da Universidade Estadual de Maringá, pelas sugestões de aperfeiçoamentos no manuscrito; a CAPES pelo suporte financeiro à pesquisa, e a Flora Essy Angeoletto, que nos estimula a escrever mais e melhor.

\section{REFERENCIAS}

AGUIAR, L.M.S.; ZORTÉA, M. A diversidade de morcegos conhecida para o Cerrado. In: SIMPÓSIO NACIONAL CERRADO/ SIMPÓSIO INTERNACIONAL SAVANAS TROPICAIS. 1, 2008, Brasília. Anais ... Planaltina: Embrapa Cerrados, 2008.

AQUINO, F.G.; MIRANDA, G.H.B. Consequências ambientais da fragmentação de habitats no Cerrado. In: Cerrado: ecologia e flora. Planaltina: Embrapa Cerrados, 2008. v. 1, p. 385-388, 2008.

ANGEOLETTO, F. Planeta ciudad: ecología urbana y planificación de ciudades medias de Brasil. Madrid, 2012. Tese (Doutorado em Ecologia e Meio Ambiente) - Universidad Autónoma de Madrid, UAM.

ANGEOLETTO, F.; SANTOS, J.W.M.C.; RUIZ SANZ, J.P. et al. Tipología socio-ambiental de las ciudades medias de Brasil: aportes para un desarrollo urbano sostenible. Urbe Revista Brasileira de Gestão Urbana, v. 8, n. 2, p. 272-287, 2016.

ANGEOLETTO, F.; RUIZ SANZ, J.P.; ALBERTIN, R.M.; SILVA, F.F. da. The grass is always greener on the other side of the fence: the flora in urban backyards of different social classes. Ambiente \& Sociedade, v. 20, n. 1, p. 1-20, 2017.

ANGEOLETTO, F.; FELLOWES, M.; SANTOS, J.W.M.C. Counting Brazil's Urban Trees Will Help Make Brazil's Urban Trees Count. Journal of Forestry, v. 116, n. 5, p. 489-490, 2018.

ANGEOLETTO, F.; LEANDRO, D.S.; FELLOWES, M. The consequences of Brazil's lack of transport planning is written in the blood of sparrows. Urban Geography, p. 1-7, Ahead of Print, 2019.

AVILA-FLORES, R.; FENTON, M.B. Use of spatial features by foraging insectivorous bats in a large urban landscape. Journal of mammalogy, v. 86, n. 6, p. 1193-1204, 2005.

BAKER, H.G. The adaptation of flowering plants to nocturnal and crepuscular pollinators. The Quarterly Review of Biology, v. 36, n. 1, p. 64-73, 1961.

BAKER, P.J.; HARRIS, S. Urban mammals: what does the future hold? An analysis of the factors affecting patterns of use of residential gardens in Great Britain. Mammal Review, v. 37, n. 4, p. 297-315, 2007.

BARCLAY, R.M.R.; BAERWALD, E.F.; GRUVER, J.C. Variation in bat and bird fatalities at wind energy facilities: assessing the effects of rotor size and tower height. Canadian Journal of Zoology, v. 85, n. 3, p. 381-387, 2007.

BARROS, R.S.M. de; BISAGGIO, E.L.; BORGES, R.C. Morcegos (Mammalia, Chiroptera) em fragmentos florestais urbanos no município de Juiz de Fora, Minas Gerais, Sudeste do Brasil. Biota Neotropica, v. 6, n. 1, p. 1-6, 2006.

BATALHA, M.A.; MANTOVANI, W. Floristic composition of the cerrado in the Pé-de-Gigante Reserve (Santa Rita do Passa Quatro, southeastern Brazil). Acta Botanica Brasilica, v. 15, n. 3, p. 289-304, 2001.

BERGALLO, H.G. et al. Bat species richness in Atlantic Forest: what is the minimum sampling effort? Biotropica, v. 35, n. 2, p. 278-288, 2003.

BERNARD, E. Vertical stratification of bat communities in primary forests of Central Amazon, Brazil. Journal of Tropical Ecology, v. 17, n. 1, p. 115-126, 2011.

BIANCONI, G.V. et al. A ecologia química da interação morcego-planta: proposta de uma nova ferramenta para restauração florestal e estudos de auto-ecologia. Morcegos do Brasil: Ecologia, Biologia e Conservação. Porto Alegre: Armazém Digital, 2008. p. 369-374. 
BORDER, J.A. et al. Predicting the likely impact of urbanisation on bat populations using citizen science data, a case study for Norfolk, UK. Landscape and Urban Planning, v. 162, p. 44-55, 2017.

BRASS, D.A. Rabies in bats. Natural History and Public Health Implications, p. 335, 1994.

BREDT, A.; UIEDA, W. Bats from urban and rural environments of the Distrito Federal, mid-western Brazil. Chiroptera Neotropical, v. 2, p. 54-57, 1996.

BREDT, A. et al. Manual de manejo e controle de morcegos em áreas urbanas e rurais. Brasília: FUNASA, 1998.

CARVALHO, C. de et al. Caracterização da fauna de morcegos (Mammalia, Chiroptera) e ocorrência de vírus rábico na região noroeste do Estado de São Paulo, Brasil. Veterinária e Zootecnia, p. 490-503, 2011.

CLERGEAU, P.et al. Bird abundance and diversity along an urban-rural gradient: a comparative study between two cities on different continents. The Condor, v. 100, n. 3, p. 413-425, 1998.

CLEVELAND, C.J. et al. Economic value of the pest control service provided by Brazilian free $\square$ tailed bats in south $\square$ central Texas. Frontiers in Ecology and the Environment, v. 4, n. 5, p. 238-243, 2006.

COLLINS, J.P. et al. A new urban ecology: modeling human communities as integral parts of ecosystems poses special problems for the development and testing of ecological theory. American scientist, v. 88, n. 5, p. 416-425, 2000.

CÔRTES V.A. et al. Abrigos diurnos e infecção rábica em morcegos de Botucatu, São Paulo, Brasil. Veterinária e Zootecnia, v. 6, p. 179-186, 1994.

COSTANZA, R. Changes in the global value of ecosystem services. Global Environmental Change, v. 26, n. 152-158, May 2014.

COUTINHO, L.M. O conceito de bioma. Acta Botanica Brasileira.v 20, n 1, p1-11, 2006.

CUNHA, E.M.S. et al. Bat rabies in the north-northwestern regions of the state of São Paulo, Brazil: 19972002. Revista de Saúde Pública, v. 40, p. 1082-1086, 2006.

DARLING, S.T.A. Protozoon general infection producing pseudotubercles in the lungs and focal necroses in the liver, spleen and lymphnodes. Journal of the American Medical Association, v. 46, n. 17, p. 1283$1285,1906$.

DEBINSKI, D.M.; HOLT, R.D. A survey and overview of habitat fragmentation experiments. Conservation Biology, v. 14, n. 2, p. 342-355, 2000.

DREW, D. Processos interativos homem-meio ambiente. 2.ed. Rio de Janeiro: Bertrand Brasil, 1989.

DURIGAN, G.; SIQUEIRA, M. F. de; FRANCO, G. A. D. C. Threats to the Cerrado remnants of the state of São Paulo, Brazil. Scientia Agricola, v. 64, n. 4, p. 355-363, 2007.

ESBÉRARD, C.E.L. Diversidade de morcegos em área de Mata Atlântica regenerada no sudeste do Brasil. Revista Brasileira de Zoociências, v. 5, n. 2, 2003.

.; BERGALLO, H.G. Coletar morcegos por seis ou doze horas a cada noite. Revista Brasileira de Zoologia, v. 22, n. 4, p. 1095-1098, 2005.

; CHAGAS, A.S.; LUZ, E.M. Uso de residências por morcegos no Estado do Rio de Janeiro (Mammalia: Chiroptera). Revista Brasileira de Medicina Veterinária, v. 21, n. 1, p. 17-20, 1999.

; BERGALLO, H.G. Research on bats in the state of Rio de Janeiro, southeastern Brazil. Mastoz. Neot., v. 12, n. 2, p. 237-243, 2005.

ESTRADA, A.; COATES-ESTRADA, R. Species composition and reproductive phenology of bats in a tropical landscape at Los Tuxtlas, Mexico. Journal of Tropical Ecology, v. 17, n. 5, p. 627-646, 2001.

FABIÁN, M.E. et al. Plantas utilizadas como alimento por morcegos (Chiroptera, Phyllostomidae), no Brasil, In: REIS, N.R.; PERACCHI, A.L.; SANTOS, G.A.S.D. (Eds). Ecologia de morcegos. Londrina: Technical Books, 2008. p. 51-70. 
et al. Alimentação de Tadarida brasiliensis (Geoffroy, 1824) na região urbana de Porto Alegre, RS, Brasil (Chiroptera, Molossidae). Revista Brasileira de Biologia, v. 50, p. 387-392, 1990.

FENTON, M.B. et al. Phyllostomid bats (Chiroptera: Phyllostomidae) as indicators of habitat disruption in the Neotropics. Biotropica, p. 440-446, 1992.

. Science and the conservation of bats. Journal of Mammalogy, v. 78, n. 1, p. 1-14, 1997.

; SIMMONS, N.B. Bats: a world of science and mystery. Chicago: University of Chicago Press, 2015.

FERREIRA, C.M.M.; FISCHER, E.; PULCHÉRIO-LEITE, A. Bat fauna in urban remnants of Cerrado in Campo Grande, Mato Grosso do Sul. Biota Neotropica, v. 10, n. 3, p. 155-160, 2010.

FERREIRA, M.S.; BORGES, A.S. Histoplasmosis. Revista da Sociedade Brasileira de Medicina Tropical, v. 42, n. 2, p. 192-198, 2009.

FRICK, W.F. et al. Fatalities at wind turbines may threaten population viability of a migratory bat. Biological Conservation, v. 209, p. 172-177, 2017.

GALINDO-GONZÁLEZ, J. et al. A more efficient technique to collect seeds dispersed by bats. Journal of Tropical Ecology, v. 25, n. 2, p. 205-209, 2009

GERMAINE, S.S.; WAKELING, B.F. Lizard species distributions and habitat occupation along an urban gradient in Tucson, Arizona, USA. Biological Conservation, v. 97, n. 2, p. 229-237, 2001.

GILBERT, O.L. Ecology of urban environment. Chapman Hall, London, 1989.

GLAESER, E. Cities, productivity, and quality of life. Science, v. 333, n. 6042, p. 592-594, 2011.

GOLDSTEIN, G. et al. Water economy of Neotropical savanna trees: six paradigms revisited. Tree Physiology, v. 28, p. 395-404, 2008.

GONÇALVES, F.; FISCHER, E.; DIRZO, R. Forest conversion to cattle ranching differentially affects taxonomic and functional groups of Neotropical bats. Biological Conservation, v. 210, p. 343-348, 2017.

GRIMM, N.B. et al. Global change and the ecology of cities. Science, v. 319, n. 5864, p. 756-760, 2008.

HARDY, P.B.; DENNIS, R.L.H. The impact of urban development on butterflies within a city region. Biodiversity \& Conservation, v. 8, n. 9, p. 1261-1279, 1999.

HICKMAN, C.P.; ROBERTS, L.S.; LARSON, A. Princípios Integrados de Zoologia. Rio de Janeiro: Guanabara Koogan, 2004.

HODSON, M.; MARVIN, S. 'Urban ecological security': a new urban paradigm? International Journal of Urban and Regional Research, v. 33, n. 1, p. 193-215, 2009.

HOORNWEG, D.; SUGAR, L.; TREJOS GÓMEZ, C.L. Cities and greenhouse gas emissions: moving forward. Environment and Urbanization, v. 23, n. 1, p. 207-227, 2011.

IBGE - Instituto Brasileiro de Geografia e Estatística. Pesquisa Nacional por Amostra de Domicílios, 2000. Disponível em: http:/ / www.ibge.gov.br. Acesso em: 18 Jan. 2019.

IBGE - Instituto Brasileiro de Geografia e Estatística. Pesquisa de Orçamentos Familiares - POF 2008-2009. 2010. Disponível em: http:/ / www.ibge.gov.br. Acesso em:18 Jan. 2019.

IBGE - Instituto Brasileiro de Geografia e Estatística. Censo Demográfico 2010. Disponível em: http:// www.ibge.gov.br. Acesso em: 18 Jan. 2019.

KAHNONITCH, I.; LUBIN, Y.; KORINE, C. Insectivorous bats in semi-arid agroecosystems- effects on foraging activity and implications for insect pest control. Agriculture, Ecosystems \& Environment, v. 261, p. 80-92, 2018.

KLINK, C.A.; MACHADO, R.B. A conservação do Cerrado brasileiro. Megadiversidade, v. 1, n. 1, p. 147155, 2005.

KRAUEL, J.J.; LEBUHN, G. Patterns of bat distribution and foraging activity in a highly urbanized temperate environment. PloS one, v. 11, n. 12, p. e0168927, 2016. 
KOOPMAN, K.F. The distributional patterns of New World nectar-feeding bats. Annals of the Missouri Botanical Garden, p. 352-369, 1981.

KOOPMAN, K.F. Order Chiroptera, p. 156-161. In: D.E. WILSON; D.M. REEDER (Eds). Mammal species of the world: a taxonomic and geografic reference. 2.ed. Washington (DC): Smithsonian Institute Press, 1993. p. 1206.

KOZLOV, M. Patterns of forest insect distribution within a large city: microlepidoptera in St Petersburg, Russia. Journal of Biogeography, v. 23, n. 1, p. 95-103, 1996.

KUNZ, T.H.; WHITAKER, J.O.; WADANOLI, M.D. Dietary energetics of the insectivorous Mexican freetailed bat (Tadarida brasiliensis) during pregnancy and lactation. Oecologia, v. 101, n. 4, p. 407-415, 1995.

KUNZ, T.H. et al. Ecological impacts of wind energy development on bats: questions, research needs, and hypotheses. Frontiers in Ecology and the Environment, v. 5, n. 6, p. 315-324, 2007.

KUNZ, T.H.; TORREZ, E.B. de; BAUER, D.et al. Ecosystem services provided by bats. Annals of the New York Academy of Sciences, n. 1223, p. 1-38, 2011.

KURTA, A.; TERAMINO, J. A. Bat community structure in an urban park. Ecography, v. 15, n. 3, p. 257-261, 1992.

LAURINDO, R.S.; NOVAES, R.L.M. Desmitificando os morcegos. Monte Belo: ISMECN, 2015.

LIMA, I.P. Espécies de morcegos (Mammalia, Chiroptera) registradas em parques nas áreas urbanas do Brasil e suas implicações no uso deste ambiente. In: REIS, N.R; PERACCHI, A.L.; Santos, G.A.S.D. (Eds). Ecologia de Morcegos. Londrina: Ed. UEL, p. 71-86, 2008.

LINLEY, G.D. The impact of artificial lighting on bats along native coastal vegetation. Australian mammalogy, v. 39, n. 2, p. 178-184, 2017.

LOBOVA, T.A. et al. Seed dispersal by bats in the Neotropics. New York: NY Botanical Garden, 2009.

LOCKWOOD, J.L.; BROOKS, T. M.; MCKINNEY, M.L. Taxonomic homogenization of the global avifauna. Animal Conservation forum, v.3, n.1, p. 27-35, 2000.

LOPEZ, J.E.; VAUGHAN, C. Observations on the role of frugivorous bats as seed dispersers in Costa Rican secondary humid forests. Acta Chiropterologica, v. 6, n. 1, p. 111-119, 2004.

MACHADO, R.B.et al. Análise de lacunas de proteção da biodiversidade no Cerrado. In: CONGRESSO BRASILEIRO DE UNIDADES DE CONSERVAÇÃO. 4, 2004, Curitiba. Anais ... p. 29-38.

MAGLE, S.B. et al. Urban wildlife research: past, present, and future. Biological conservation, v. 155, p. 23-32, 2012.

MARINHO-FILHO, J. Distribution of bat diversity in the southern and southeastern Brazilian Atlantic Forest. Chiropt. Neotrop., v. 2, n. 2, p. 51-54, 1996.

MARQUES, R.V.; FABIÁN, M.E. Ciclo reprodutivo de Tadarida brasiliensis (I. Geoffroy, 1824)(Chiroptera, Molossidae) em Porto Alegre, Brasil. Iheringia, Série Zoologia, v. 77, p. 45-56, 1994.

MARQUES, R.V.; PACHECO, S.M. Comportamento de cópula de Noctilio leporinus (Linnaeus, 1758) (Mammalia, Chiroptera, Noctilionidae). Comunicações do Museu de Ciências e Tecnologia PUCRS, zool, v. 12, p. 193-200, 1999.

MARTINS, R.L.; GRIBEL, R. Polinização de Caryocar villosum (Aubl.) Pers.(Caryocaraceae) uma árvore emergente da Amazônia Central. Revista Brasileira de Botânica, v. 30, n. 1, p. 37-45, 2007.

McCRACKEN, G.F. et al. High altitude foraging by Brazilian free-tailed bats Tadarida brasiliensis on migratory insect pests. Bat Research News, n. 42, p. 110, 2001.

McDONALD, R.I.; MARTY, K.L.; FORMAN, R.T.T. The implications of current and future urbanization for global protected areas and biodiversity conservation. Biol. Conservation, v.141, p. 1695-1703, 2008.

McKINNEY, M.L.; LOCKWOOD, J. L. Biotic homogenization: a few winners replacing many losers in the next mass extinction. Trends in ecology \& evolution, v. 14, n. 11, p. 450-453, 1999. 
McKINNEY, M.L. Urbanization, biodiversity, and conservation. Bioscience 52: 883890McKinney ML (2006) Urbanization as a major cause of biotic homogenization. Biol Conserv, v. 127, p. 247260, 2002.

McKINNEY, M.L. Effects of urbanization on species richness: a review of plants and animals. Urban ecosystems, v. 11, n. 2, p. 161-176, 2008.

MEDELLÍN, R.A.; EQUIHUA, M.; AMIN, M.A. Bat diversity and abundance as indicators of disturbance in Neotropical rainforests. Conservation biology, v. 14, n. 6, p. 1666-1675, 2000.

MUSCARELLA, R.; FLEMING, T.H. The role of frugivorous bats in tropical forest succession. Biological reviews, v. 82, n. 4, p. 573-590, 2007.

MYERS, N. et al. Biodiversity hotspots for conservation priorities. Nature, v. 403, n. 6772, p. 853, 2000.

NEGRI, S. Modernização agrícola e reorganização do espaço agrário da mesorregião sudeste matogrossense. Uberlândia, 2001. Dissertação (Mestrado em Geografia) - Universidade Federal de Uberlândia, UFU.

NEGRI, S. O processo de segregação sócio-espacial no contexto do desenvolvimento econômico da cidade de Rondonópolis-MT. Rio Claro, 2008. Tese (Doutorado em Geografia) - Universidade Estadual Paulista Julio de Mesquita Filho, UNESP.

NEUWEILER, G. The Biology of Bats. New York: Oxford University Press, 2000.

NEWMAN, P. The environmental impact of cities. Environment and Urbanization, v. 18, n. 2, p. 275-295, 2006.

NOGUEIRA, M. et al. Checklist of Brazilian bats, with comments on original records. Check list, v. 10, p. 808, 2014.

NUNES, H.; ROCHA, F.L.; CORDEIRO-ESTRELA, P. Bats in urban areas of Brazil: roosts, food resources and parasites in disturbed environments. Urban ecosystems, v. 20, n. 4, p. 953-969, 2017.

Oliveira, H.F.M.; Camargo, N.F.; Gager, Y. et al. Protecting the Cerrado: where should we direct efforts for the conservation of bat-plant interactions? Biodiversity and Conservation, 2019, In Press.

OPREA, M. et al. Do wooded streets provide connectivity for bats in an urban landscape? Biodiversity and Conservation, v. 18, n. 9, p. 2361-2371, 2009.

PACHECO, S.M. et al. Morcegos urbanos: status do conhecimento e plano de ação para a conservação no Brasil. Chiroptera Neotropical, v. 16, n. 1, p. 629-647, 2010.

PEDRO, W.A. Morcegos na área urbana. Biológico, v.2, n.60, p. 101-102,1998.

PERACCHI, A.L. et al. Ordem Chiroptera. In: REIS, N.R.et al. (Ed.). Mamíferos do Brasil. Londrina: Ed. UEL, p.153-230, 2006.

PERACCHI, A.L. NOGUEIRA, M.R. Lista anotada dos morcegos do Estado do Rio de Janeiro, Sudeste do Brasil. Chiroptera Neotropical, v. 16, n. 1, p. 508-519, 2010.

PERINI, F.A.; TAVARES, V.C.; NASCIMENTO, C. Bats from the city of Belo Horizonte, Minas Gerais, southeastern Brazil. Chiroptera Neotropical, v. 9, n. 1-2, p. 169-173, 2014.

REIS, N.R. et al. (Ed.). Morcegos do Brasil. Londrina: Ed. UEL, 2007.

REIS, N.R.; DE LIMA, I.P.; PERACCHI, A.L. Morcegos (Chiroptera) da área urbana de Londrina, Paraná, Brasil. Rev Bras Zool, v. 19, p. 739-746, 2006.

REIS, N.R.; LIMA, I.P.; PERACCHI, A.L. et al. Ordem Chiroptera. In: REIS, N.R.; LIMA, I.P.; PERACCHI, A.L.; PEDRO W.A. (Orgs.). Mamíferos do Brasil. Londrina: Ed. UEL, p.153-230. 2006

RODRÍGUEZ-AGUILAR, G.et al. Influence of urbanization on the occurrence and activity of aerial insectivorous bats. Urban Ecosystems, v. 20, n. 2, p. 477-488, 2017.

RUMBLE, H.; ANGEOLETTO, F.; CONNOP, S. et al. Understanding and applying ecological principles in cities. In LEMES DE OLIVEIRA, F.; MELL, I (Eds.). Planning Cities with Nature: Theories, Strategies and Methods. Amsterdam: Springer Nature, 2019. 
RYDELL, J.; RACEY, P.A. Street lamps and the feeding ecology of insectivorous bats. In: Symposia of the Zoological Society of London. London: The Zoological Society, 1995. p. 291-307.

SAMPAIO, E.M. et al. A biodiversity assessment of bats (Chiroptera) in a tropical lowland rainforest of Central Amazonia, including methodological and conservation considerations. Studies on Neotropical fauna and environment, v. 38, n. 1, p. 17-31, 2003.

SANTOS, M. A urbanização brasileira. São Paulo: Ed. USP, 2005.

SATO, T. M.; PASSOS, F. De C.; NOGUEIRA, A.C. Frugivoria de morcegos (Mammalia, Chiroptera) em Cecropia pachystachya (Urticaceae) e seus efeitos na germinação das sementes. Papéis Avulsos de Zoologia, v. 48, n. 3, p. 19-26, 2008.

SAZIMA, M.; BUZATO, S.; SAZIMA, I. Dyssochroma viridiflorum (Solanaceae): a reproductively bat $\square$ dependent epiphyte from the Atlantic Rainforest in Brazil. Annals of Botany, v. 92, n. 5, p. 725-730, 2003.

SILVA, M.M.; HARMANI, N.M.S.; GONÇALVES, E.F.B; UIEDA, W. Bats from the metropolitan region of São Paulo, southeastern Brazil. Chiroptera Neotropical, v. 2, n. 1, p. 39-41, 1996.

Silva, N.M.; Angeoletto, F.; Santos, J.W.M.C. et al. The negative influences of the new Brazilian forest code on the conservation of riparian forests. European Journal of Ecology, v.3, n 2, p. 116-122, 2017.

SILVA, R.; PERINI, F.A.; OLIVEIRA, W.R. Bats from the city of Itabira, Minas Gerais, southeastern Brazil. Chiroptera Neotropical, v. 11, n. 1-2, p. 216-219, 2005.

SILVA, S.S.; PERACCHI, A.L. Observation of visit of bats (Chiroptera) to the flowers of Pseudobombax grandiflorum (Cav.) A. Robyns. Revista Brasileira de Zoologia, v. 12, n. 4, p. 859-865, 1995.

SIMMONS, N.B. An Eocene big bang for bats. Science, v. 307, n. 5709, p. 527-528, 2005.

SOARES, S.C. et al. Percepção dos Moradores de Goioerê-PR, sobre a Fauna Silvestre Urbana. Arquivos do MUDI, v. 15, p. 1, 2011.

SODRÉ, M.; ESBÉRARD, C.E.L. Morcegos Urbanos do Sudeste do Brasil. In: PACHECO, S.M.; MARQUES, R.V.; ESBÉRARD, C.E.L. (Orgs.). Morcegos no Brasil: Biologia, Sistemática, Ecologia e Conservação. Porto Alegre: Armazém Digital, 2008. p. 407- 414.

SODRÉ, M.; GAMA, A.R.; ALMEIDA, M.F. Updated list of bat species positive for rabies in Brazil. Revista Instituto de Medicina Tropical,v. 52, p. 75-81, 2010.

TADDEI, V.A. Morcegos: algumas considerações sistemáticas e biológicas. Campinas, CATI, 1983. (Boletim técnico, 172).

TIRIRA, D. Historia Natural de los murciélagos Neotrópicales. Biología, Sistemática y Conservación de los mamíferos del Ecuador. Museo de Zoología, Centro de Biodiversidad y Ambiente. Pontificia Universidad Católica del Ecuador, v. 1, p. 31-56, 1998.

THIES, W. KALKO, E.K.V. Phenology of neotropical pepper plants (Piperaceae) and their association with their main dispersers, two short-tailed fruit bats, Carollia perspicillata and C. castanea (Phyllostomidae). Oikos, v. 104, n. 2, p. 362-376, 2004.

TRENTINI, R.P. Fatores antrópico-ambientais determinantes para o aumento de acidentes loxoscélicos no município de Curitiba-Paraná. Curitiba, 2005. Dissertação (Mestrado em Geografia) - Universidade Federal do Paraná, UFPR.

TSANG, S.M. et al. The roles of taxonomy and systematics in bat conservation. In: VOIGT, C.C.; KINGSTON, T. (Eds.) Bats in the Anthropocene: Conservation of bats in a changing world. London: Springer Cham, 2016. p. 503-538.

UIEDA, W. HARMANI, N.; SILVA, M. Raiva em morcegos insetívoros (Molossidae) do Sudeste do Brasil. Revista de Saúde Pública, v. 29, p. 393-397, 1995.

Vacchiano, M.C.; Santos, J.W.M.C.; Angeoletto, F.; Silva, N.M. do Data Support Claims That Brazil Leads the World in Environmental Preservation? Environmental Conservation, v. 46, n. 2, p. 118-120, 2019. 
VERDE, R.S.; SILVA, R.C.; CALOURO, A.M. Activity patterns of frugivorous phyllostomid bats in an urban fragment in southwest Amazonia, Brazil. Iheringia. Série Zoologica, v. 108, p. 1-7, 2018.

VILHENA-VIALOU, A.V. Pré-história do Mato Grosso. São Paulo: Ed. USP, 2006. v.2: Cidade de Pedra.

ZORTÉA, M.; AGUIAR, L. Foraging behavior of the fishing bat, Noctilio leporinus (Noctilionidae). Chiroptera Neotropical, v. 7, n. 1-2, p. 140-142, 2001.

WILSON, M.F. Dispersal of seeds by frugivorous animals in temperate forests. Revista Chilena de Historia Natural, v. 64, n. 3, p. 537-554, 1991.

WORDLEY, C.F.R. et al. Bats in the Ghats: Agricultural intensification reduces functional diversity and increases trait filtering in a biodiversity hotspot in India. Biological conservation, v. 210, p. 48-55, 2017.

Data de submissão: 23/ mar./ 2019

Data de aceite: 22/ jul./ 2019 